\title{
添加氢氧化铝的铁铬催化剂的 表面性质和物性研究
}

张惠 良

陈 文 锋

（南京大学化学系,南京 210008）（南京化学工业公司化工仾究院, 湳京 210008）

胡 梅 生

（南京大学现代分析中心，南京 210008）

\section{关链铁催化剂、氢氧化铝的影响、表面性质和物性}

我们曾报道了添加氢氧化铝的铁铬催化剂的穆斯堡尔谱及磁性研究 ${ }^{[1]}$. 本文采用了 X射 线光电子能谱 (XPS) 和透射电镜 (TEM) 及微区元亚分析, 继续考察了 $\mathrm{Al}(\mathrm{OH})_{3}$ 添加后, 对 催化剂的表面性质和物性的影响.

\section{一、实验部分}

1. 样品制备与测定样品制备、活性、比表面积、机诚强度测定见文献 [1].

2. XPS 测定 采用 $\mathrm{PHI} / 550$ 型电子能谱仪, $\mathrm{AlK} a$ 为 $\mathrm{X}$ 光源, 功率 $400 \mathrm{~W}$. 样品表面 电荷效应采用污染 $\mathrm{C}_{1 s}$ 结合能 $(284.6 \mathrm{eV})$ 为标准进行校正, 仪器误差 $\pm 0.3 \mathrm{eV}$.

3. TEM 及微区元素分析样品用蒸馏水分散, 沉积在真空喷镀过碳的火棉胶铜网上, 用 $\mathrm{JEM}-200 \mathrm{CX}$ 型，高压 $200 \mathrm{kV}$ 下拍摄. 在相同条件下，用石墨样品台，进行微区元素分 析.

\section{二、结果和讨论}

表 1 为测定中所采用的三种售化剂样品的化学分析、Al $(\mathrm{OH})_{3}$ 添加量、比表面积、活性和 比械强度等测定结果. 可以看到, $\mathrm{Al}(\mathrm{OH})_{3}$ 添加后, 样品的比表面积和机栰强度增大, 活性基

表 1 三个样品的化学分析、催化活性、比表面积和机械强度测定

\begin{tabular}{|c|c|c|c|c|c|}
\hline 样品编号 & $\begin{array}{c}\text { 添加 } \\
\frac{\mathrm{Al}(\mathrm{OH})_{3}}{\mathrm{Fe}_{2} \mathrm{O}_{3}} \\
(\mathrm{wt} \%)\end{array}$ & $\begin{array}{c}\text { 化学分析 } \\
\mathrm{CrO}_{3} \\
\mathrm{Fe}_{2} \mathrm{O}_{3} \\
(w t \%)\end{array}$ & $\begin{array}{c}\text { 比表面积 } \\
\left(\mathrm{m}^{2} / \mathrm{g}\right)\end{array}$ & $\begin{array}{l}\text { 婪械强度 } \\
\left(\mathrm{kg} / \mathrm{cm}^{2}\right)\end{array}$ & $\begin{array}{c}\text { 催化活性, 空逨 } 500 \mathrm{~h}^{-1} \\
\left.\text { (经 } 500^{\circ} \mathrm{C} \text { 耐热 } 5 \mathrm{~h}\right)\end{array}$ \\
\hline $\mathbf{A}$ & 0.0 & 17.12 & 90.4 & 195 & 93.4 \\
\hline c & 1.0 & 16.94 & 100 & 287 & 92.8 \\
\hline E & 2.0 & 16.84 & 111 & 274 & 92.4 \\
\hline
\end{tabular}

本文 1988 年 12 月 20 日收到. 1989 年 7 月 23 日收到修改稿. 


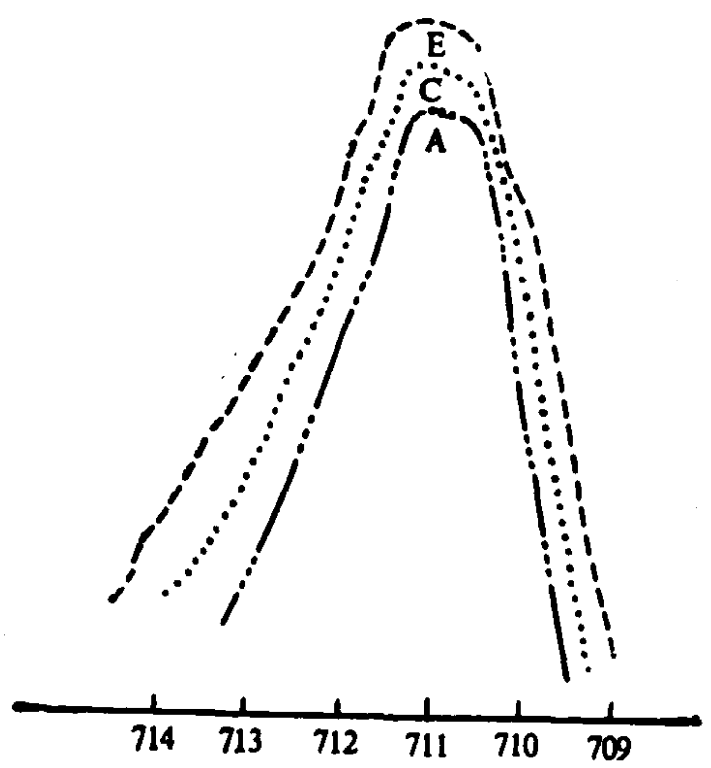

结合触(eV)

a. $\mathrm{Fe}_{213 / 2}$ 的 XPS 因谓

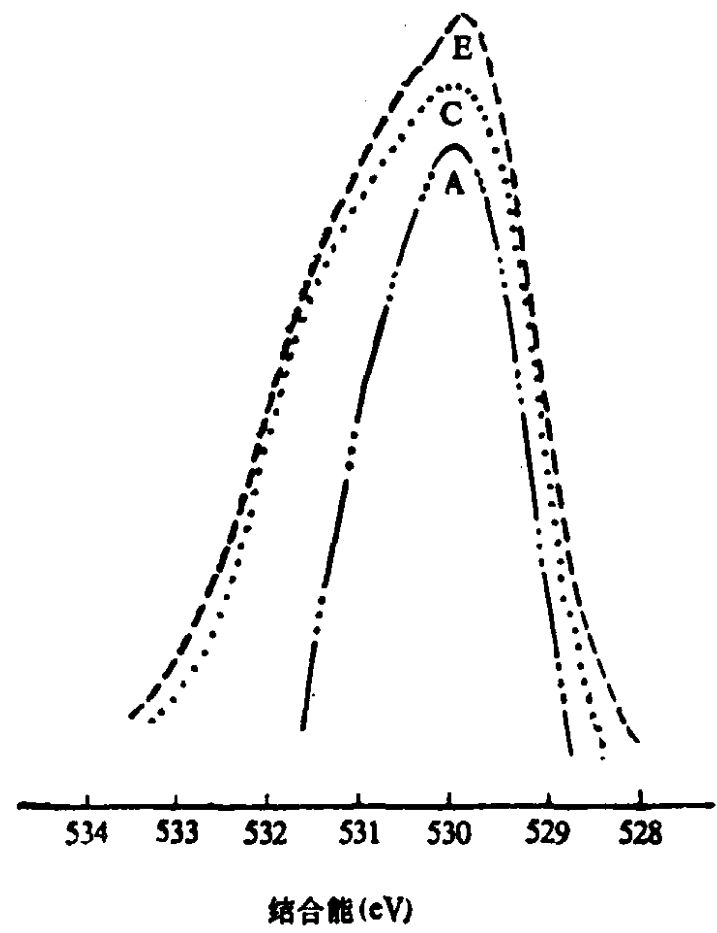

c. $\mathrm{O}_{1}$ 的 XPS 图谱

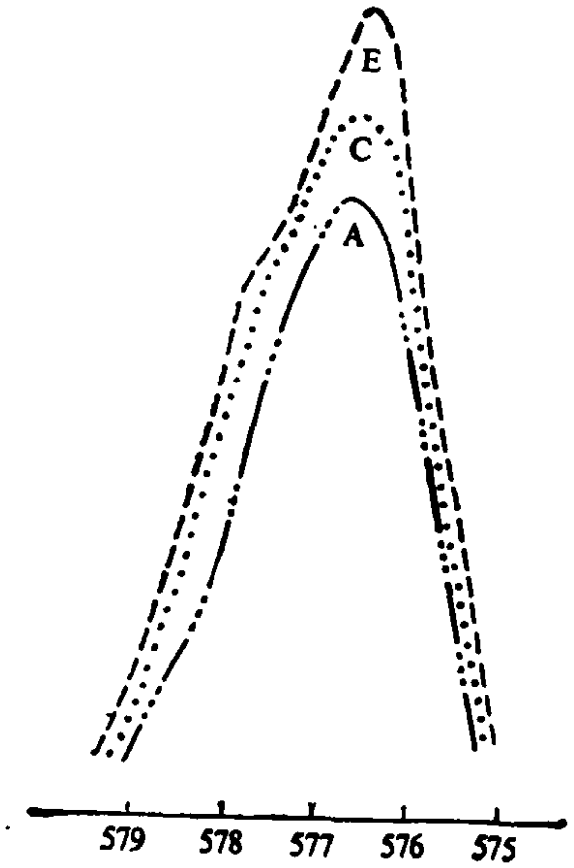

结合能(cV)

b. $\mathrm{Cr}_{2 P 3 / 3}$ 的 XPS 因徣

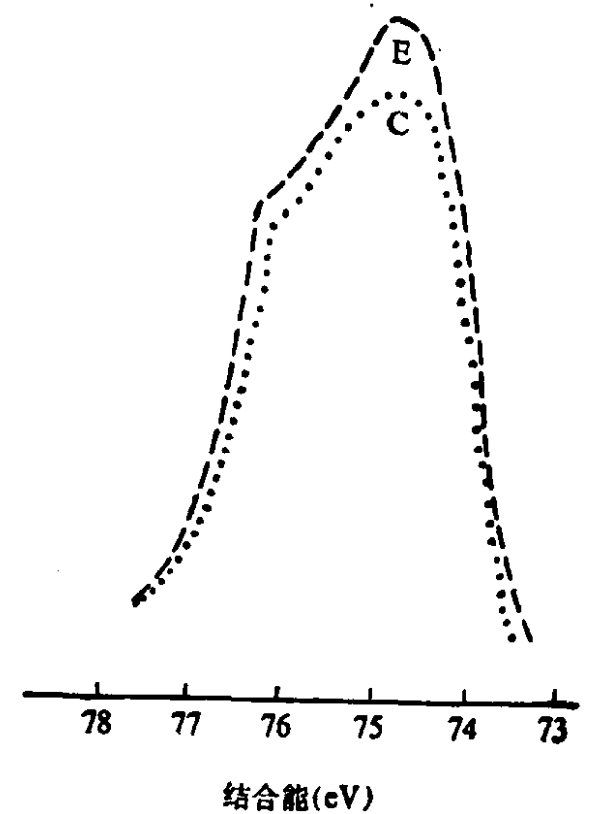

d. $\mathrm{Al}_{2 p}$ 的 XPS 图徣

图 $1 A 、 C 、 E$ 样品的 XPS 图谱

本上保持不变. 图 1 是 XPS 结果. $\mathrm{Fe}_{2,3 / 2}$ 的结合能谱图(见图 $1 \mathrm{a}$ ), 峰较宽并较为不规则, 表 明峰形相互叠加，铁在表面上存在有几种化学奖态。根据文献[2]报道, $\mathrm{Fe}_{3} \mathrm{O}_{4}$ 的 $\mathrm{Fe}_{2 \mathrm{P} 3 / 2}$ 结合 能为 $711.3 \mathrm{eV}, \mathrm{Fe}_{2} \mathrm{O}_{3}$ 的 $\mathrm{Fe}_{2 p^{3 / 2} / 2}$ 结合能为 $710.5 \mathrm{eV}$, 以及实验测得铁铬固溶体的 $\mathrm{Fe}_{2 p^{3} / 2}$ 结合能 为 $709.7 \mathrm{eV}$ 和 XRD 结果 ${ }^{[1]}$, 表明在样品表面上, 铁以 $\mathrm{Fe}_{2} \mathrm{O}_{3} 、 \mathrm{Fe}_{3} \mathrm{O}_{4} 、(\mathrm{Fe} 、 \mathrm{Cr})_{2} \mathrm{O}_{3}$ 化合物等存 
在. 图 $1 \mathrm{~b}$ 为 $\mathrm{Cr}_{2,3 / 2}$ 的结合能谱图, 其峰形也较宽, 从报道的结合能数值, $\mathrm{Cr}_{2} \mathrm{O}_{3}$ 为 $576.6 \mathrm{eV}$, $\mathrm{CrO}_{3}$ 为 $578.1 \mathrm{eV}$ 表明存在有 $\mathrm{Cr}_{2} \mathrm{O}_{3} 、(\mathrm{Fe}, \mathrm{Cr})_{2} \mathrm{O}$ 、和含量较低的 $\mathrm{CrO}$, 等含 $\mathrm{Cr}$ 的化合物. 图 Ic 为 $\mathrm{O}_{1}$ 能级结合能谱图, 未添加 $\mathrm{Al}(\mathrm{OH})$, 的样.品 $\mathrm{A}$, 其峰形较窄, $\mathrm{Al}(\mathrm{OH})_{3}$ 添加后, 蜂形增 宽, 当添加量为 $2 \%$ 时, 峰形最宽, 表明 $\mathrm{Al}(\mathrm{OH})_{3}$ 添加后, 催化剂表面存在有不同的化学状态 的化物. 根据 $\mathrm{Al}_{2} \mathrm{O}_{3}$ 的 $\mathrm{O}_{1 s}$ 的结合能值为 $531.6 \mathrm{eV}, \mathrm{Cr}_{2} \mathrm{O}_{3}$ 的 $\mathrm{O}_{18}$ 的结合能值为 $530.3 \mathrm{eV}$, 而 $\mathrm{Fe}_{2} \mathrm{O}_{3}$ 的 $\mathrm{O}_{\mathrm{u}}$ 结合能为 $530 \mathrm{eV}$, 结合上述图 $1 \mathrm{a}$ 和 $1 \mathrm{~b}$ 的结果, 可以认为在 $\mathrm{C} 、 \mathrm{E}$ 样品表面上是 以图 $1 \mathrm{a}$ 和 $1 \mathrm{~b}$ 中指出的 $\mathrm{Al}_{2} \mathrm{O}_{3} 、 \mathrm{Cr}_{2} \mathrm{O}_{3} 、 \mathrm{Fe}_{2} \mathrm{O}_{3}$ 等氧化物的形式存在. 图 $1 \mathrm{~d}$ 为添加有 $\mathrm{Al}(\mathrm{OH})$, 样品 $\mathrm{C} 、 \mathrm{E}$ 的 $\mathrm{Al}_{2 \mathrm{p}}$ 能级结合能图谱, 可以看到, 除在 $74.7 \mathrm{eV}$ 处为 $\mathrm{Al}_{2} \mathrm{O}_{3}$ 的结合能值外, 在结合 能为 $76.3 \mathrm{eV}$ 处均出现有肩峰. 根据 $\mathrm{Al}(\mathrm{OH})_{3}$ 的 $\mathrm{O}_{1}$ 结合能比 $\mathrm{Al}_{2} \mathrm{O}_{3}$ 的 $\mathrm{O}_{1}$ 结合能高 $1.6 \mathrm{eV}^{[2]}$, 该肩峰对 $\mathrm{Al}_{2} \mathrm{O}_{3}$ 的结合能的化学位移值为 $1.6 \mathrm{eV}$, 同 $\mathrm{Barrie}^{[3]}$ 报道的 $\mathrm{AlO}_{x}$ 的结合能为 $75.3 \mathrm{eV}$ 以及该肩峰结合能对 $\mathrm{AlO}_{x}$ 的化学位移数值为 $1.0 \mathrm{eV}$ 等认识, 对该肩峰可指属为在样品表面上 存在的少量 $\mathrm{AlO}_{2}^{-}$(或 $\mathrm{AlO}_{3}$ ) 化合物. 样品 $\mathrm{C}$ 和 $\mathrm{E}$ 均进行了 $\mathrm{Al} 、 \mathrm{Cr} 、 \mathrm{C} 、 \mathrm{O}$ 及 $\mathrm{Fe}$ 等原子的相对 浓度对 $\mathrm{Ar}^{+}$离子的贼射时间测定, 剥离原度 $400 \AA$. 测定表明: 在样品的外表面上碳的富 集量 $(25.1 \%)$ 比表面层内的富集量 $(11.4 \%)$ 高; 铁的含量相反, 表面层外含量 $(11.2 \%)$ 比表 面层内 $(21.9 \%)$ 低; 铬的含量由 $7.7 \%$ 改变到 $8.1 \%$, 仅改变 $0.4 \%$, 变化很少; 氧的含量随諳 $\mathrm{Ar}^{+}$践射时间增大变化不大; 铝含量基本恒定, 表明样品表面经 $\mathrm{Ar}^{+}$的轰击剥离后, 在 XPS 的 探测线度为 $250 \mu \mathrm{m}$ 的范围内, 铝是均匀的分布, 考虑添加的 $\mathrm{Al}(\mathrm{OH})_{3}$ 是以胶粒形式包围着催 化剂微粒表面, 并相互连接组成了粒子的集合体, 当经 $\mathrm{Ar}^{+}$离子由外表面往里层逐次戝射剥 离时, 测到的 $\mathrm{Al}$ 含昷应是恒定的平均数值.

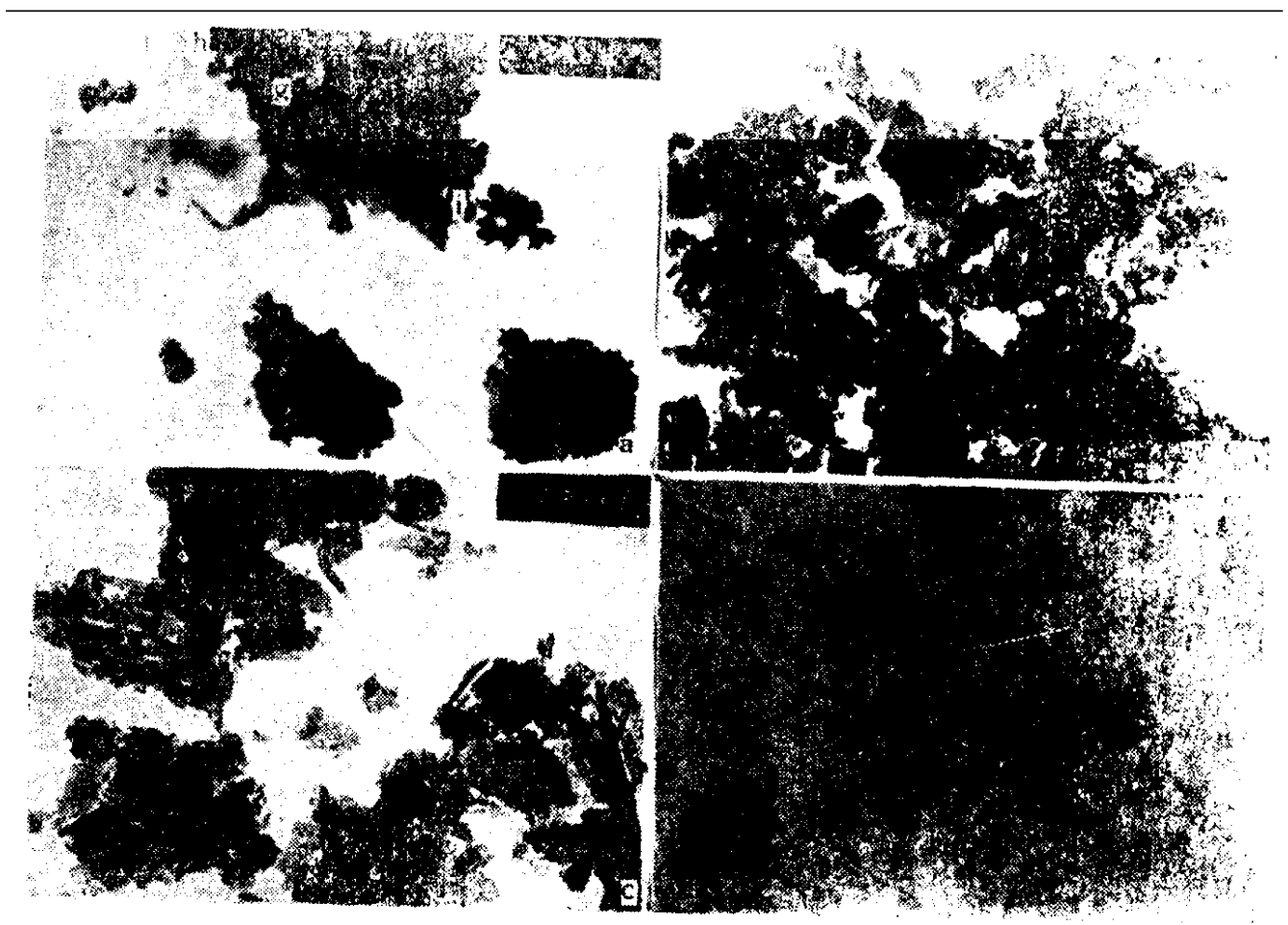

图 2 TEM 样品的照序

a. 样品 E的 TEM 炤片 $(\times 10500)$; b. 样品E的 TEM 照片 $(\times 35000) ;$ c. 样品 E 的 TEM 照片 $(\times 70000) ;$ d. 样品 A 的 TEM 照片 $(\times 25900)$

图 2 是样品的. TEM 昭片. 图 $2 \mathrm{a} 、 \mathrm{~b} 、 \mathrm{c}$ 为添加有 $\mathrm{Al}(\mathrm{OH})_{3}$ 的样品 $\mathrm{E}$ 的不同放大倍数的 TEM 照片, 其外形以圆粒状较多, 长棒状较少, 而未添加 $\mathrm{Al}(\mathrm{OH})$ ）的样品的图 $2 \mathrm{~d}$, 明显以针、 
棒等形状占多数. 因此表明 $\mathrm{Al}(\mathrm{OH})$ ：添加后，催化剂的形貌有所改变. 从样品 $\mathrm{E}$ 另一分散较好 的粒子图像, 取 30 个粒子数, 其平均大小为 $0.01 \mathrm{~cm}$, 除以照片的放大倍数 (10500), 得到粒子 平均大小为 $66.7 \AA$, 这一结果与报道 ${ }^{\text {[) }}$ 的计算值吻合. 由图 2a TEM 照片中选择了 $\mathrm{g} 、 \mathrm{~h} 、 \mathrm{i} \equiv$ 个点区, 进行了 $\mathrm{Fe} 、 \mathrm{Cr} 、 \mathrm{Al}$ 含量的微区分析, 测定的结果是以 $\mathrm{Fe}$ 的满标刻度 (100\%) 为基准 计算求得, 表明在 $\mathrm{i}$ 点处没有 $\mathrm{Al}(0.0 \%), \mathrm{h}$ 点处 $\mathrm{Al}$ 的含量较低 $(9.1 \%), \mathrm{g}$ 处 $\mathrm{Al}$ 的含量最高 (30.5\%), 即在 TEM 的 $500 \AA$ 探测线度测定的样品的表面微区中, 铝的分布是不均匀的. 这 一结果和 XPS 所得的 Al 含量随 $\mathrm{Ar}^{+}$茂射剥离的测定不矛盾, 因为在 XPS 中测量的线度为 $250 \mu \mathrm{m}$ ，是 TEM 测量的 $500 \AA$ 线度的 5000 倍, 因此大面积下测定的 $\mathrm{Al}$ 量应是恒定值. 而测 定的 $\mathrm{Cr}$ 含量较为一致, $\mathrm{Cr}$ 多以铁铬固溶体存在, $\mathrm{Cr}$ 进入氧化铁晶格, 故测得的 $\mathrm{Cr}$ 含量较为 恒定. 因此, 以 $\mathrm{Al}$ 和 $\mathrm{Cr}$ 的微区分析结果对比, $\mathrm{Al}^{3+}$ 未进入氧化铁晶格, 如果 $\mathrm{Al}^{3+}$ 进入氧化 铁晶格，则 TEM 微区分析所测得的 $\mathrm{Al}$ 含量应基本上近似为定值, 所以 TEM 结果和文献 [1] 的穆斯堡尔谱测定结果是一致的. 添加的铝是以氧化物形式分布在铁铬氧化物微粒的表 面 上,由于铝的不均匀分布, 铝未能遮盖 $\mathrm{CO}$ 变换反应中的 $\mathrm{Fe}_{3} \mathrm{O}_{4}$ 活性部位, 因而当 $\mathrm{Al}(\mathrm{OH})_{3}$ 添 加量为 $2 \%$ 时, 样品 $\mathrm{E}$ 的活性仍很高, 和未添加 $\mathrm{Al}(\mathrm{OH})_{3}$ 的样品相比基本一致, 实验也得到, 当 $\mathrm{Al}(\mathrm{OH})$ ，含量 $>6 \%$ 时，催化活性有明显下降。这一在微粒外表面上覆盖的 $\mathrm{Al}(\mathrm{OH})$ ， 经制备中的加热和焙烧, 与锌化铁等氧化物进一步相互作用脱水后, 形成了 $\mathrm{AlO}_{2}^{-}\left(\mathrm{AlO}_{3}\right)$ 形 式的铝氧桥联结构, 以及由于保持了催化剂微粒的聚集性等特征, 从而使样品的强度和比表面 积都明显增大。

\section{四、结论}

$\mathrm{Al}(\mathrm{OH})_{3}$ 源加后, 使样品中微粒的形貌改变, 多为圆粒状. 加人的 $\mathrm{Al}(\mathrm{OH})_{3}$ 和 $\mathrm{CrO}_{3}$ 不 同, 铬进人催化剂微粒的晶格, 而铝是以 $\mathrm{Al}_{2} \mathrm{O}_{3}$ 和 $\mathrm{AlO}_{2}^{-}$(或 $\mathrm{AlO}_{3}$ ) 的形式不均匀的分布在微 粒的外表面上.

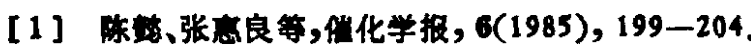

[2] Wagner, C. D. et al., Handbook of X-Ray Photoelectron Spectroscopy, Perkin Elmer, Eden Prairie= 1979.

[3] Barrie, A., Chem. Phys. Letr., 19(1973), 109. 